\title{
Valoração Contingente do Parque "Chico Mendes": uma Aplicação Probabilística do Método Referendum com Bidding Games*
}

\author{
Rubicleis Gomes da Silva ${ }^{1}$ \\ João Eustáquio de Lima²
}

Resumo: A valoração ambiental é uma ferramenta relevante para determinação do valor de bens que não possuem mercado definido. O parque ambiental "Chico Mendes", situado em Rio Branco - Acre, em razão de suas características de não-rivalidade e não-excludência, é um bem público. O método de valoração contingente constitui uma das técnicas que possibilitam a determinação do valor econômico de um bem público. De forma geral, este trabalho busca determinar o valor econômico total do parque "Chico Mendes". Os resultados mostram que a renda familiar, o valor da verdadeira disposição a pagar, o sexo e a idade são variáveis importantes para explicar probabilidade do indivíduo se dispor a contribuir para conservação e manutenção dos serviços oferecidos pelo parque. Por fim, o bootstrapping e a análise de risco fornecem maior segurança aos resultados encontrados.

\footnotetext{
* Artigo extraído da Dissertação de Mestrado do primeiro autor, orientada pelo segundo. Vencedora do Prêmio EDWARD SHUCH, 2003, concedido pela Sociedade Brasileira de Economia e Sociologia Rural (SOBER) de melhor Tese de Mestrado em Economia. Os autores agradecem à CAPES e à Universidade Federal do Acre (UFAC) pelo financiamento desta pesquisa.

${ }^{1}$ Servidor da UFAC, Economista, Mestre em Economia Aplicada e Doutorando em Economia Aplicada pelo Departamento de Economia Rural (DER) da Universidade Federal de Viçosa.rubicleis@uol.com.br

${ }^{2}$ Engenheiro Agrônomo, Mestre em Economia Rural, Ph.D., em Economia Rural e Professor Titular do DER/UFV, Viçosa - MG. jelima@ufu.br
} 
Palavras-Chave: Valoração ambiental (contingente), bens públicos, modelos dicotômicos, bootstrapping.

Classificação JEL: Q51, H41, C35.

Abstract: The object of this study is the environmental park "Chico Mendes" located in Rio Branco - Acre. Being unique and all-enclosing in its nature it is a public asset. The contingent valuation makes possible the determination of the total economic value total of a public asset. The people's willingness to pay for the park maintenance and conservation shows a determination of the consumers' preference. This work attempts to determine the total economic value of the park. The results show that family income, value of monthly contribution, sex and age explain the probability of a person to contribute to the park conservation. Finally, bootstrapping and risk analysis provide greater safety to the results found.

Key-Words: Contingent valuation, publics assets, dichotomous choice, bootstrapping

JEL Classification: Q51, H41, C35.

\section{Introdução}

\subsection{Considerações iniciais}

A utilização intensiva dos recursos naturais renováveis e não-renováveis trouxeram à sociedade a preocupação com a inter-relação existente entre crescimento e desenvolvimento econômico com o meio ambiente. Consequentemente, surgiu a necessidade de conciliar variáveis econômicas com a questão ambiental, com o intuito de tornar possível um relacionamento harmônico entre as necessidades humanas e as disponibilidades ambientais.

Nesse contexto, a valoração de ativos ambientais busca sinalizar o preço que um recurso ambiental possui, tornando possível a determinação de políticas que visem conciliar a manutenção e conservação do meio ambiente, conjuntamente, com as necessidades humanas e econômicas. 
O objeto de pesquisa deste trabalho é o parque ambiental "Chico Mendes” (PACM), que está situado na Rodovia AC-40, aproximadamente a $10 \mathrm{~km}$ do centro de Rio Branco, capital do estado do Acre, o único parque ambiental presente no município. Possui cerca de 52 hectares, 50\% (28,0 ha) dos quais são cobertos de floresta primária, sendo o restante da área dividida em duas partes; a mata secundária alta, que cobre $22 \%$ (11,5 ha) da área total, e os restantes 27,7\% (14,5 ha), onde já há algum tipo de ação entrópica. (SEMEIA, 1996)

A floresta pertencente à área do PACM apresenta uma biodiversidade elevada, com espécies de alto valor econômico, biológico e medicinal. Em 1998, 50 mil pessoas visitaram o parque, perfazendo um total aproximado de $10 \%$ da população do estado e, aproximadamente, $20 \%$ da população do município de Rio Branco (SEMEIA, 1996).

Como PACM é um bem público, mais precisamente um ativo ambiental, a disposição a pagar pela conservação e manutenção desse ativo não indica somente um valor que reflete as preferências dos indivíduos, mais possibilita averiguar o grau de conscientização da sociedade acerca da preservação de um recurso ambiental. Nesse contexto, é importante verificar se o indivíduo possui a percepção de que danos ou melhorias em bens ambientais possam se manifestar em acréscimos ou decréscimo em seu bem-estar.

Conforme SILVA (2003), a importância da valoração ambiental reside no fato de que esta é essencial para criar um valor de referência que indique uma sinalização de mercado, possibilitando, assim, o uso "racional" dos recursos ambientais. Com isso, os agentes públicos e privados terão subsídios para avaliação econômica de tomadas de decisões políticas sobre a utilização eficiente desses ativos. Logo, a criação de um valor de referência para um bem ambiental fornece informações ao poder público, à sociedade civil organizada e às organizações nãogovernamentais (ONGs), possibilitando um gerenciamento mais eficaz desses recursos.

O objetivo central desta pesquisa consiste em verificar se a sociedade rio-branquense possui a percepção de que a manutenção e conservação do PACM aumentam o seu nível de bem-estar. Especificamente, pretendese: a) Estimar o valor econômico do Parque Ambiental "Chico Mendes"; b) Estimar e analisar a disposição a pagar manifestada (stated willingness 
- to-pay) e a verdadeira disposição a pagar (true willingness - to-pay) para manutenção e conservação do parque e; c) Efetuar uma análise de risco sobre a verdadeira disposição a pagar.

No Brasil diversos estudos utilizaram a avaliação contingente para determinar a disposição a pagar pela conservação e manutenção de um bem ou serviço ambiental. Dentre eles, podem-se citar os de: RIBEIRO (1998), que estimou a disposição a pagar pela despoluição do rio Meia Ponte em Goiânia; BELLUZZO JR. (1995), estimou a disposição a pagar pela despoluição do rio Tiête; AGUIRRE e FARIA (1996), que estimaram os benefícios oriundos da despoluição da principal área urbana do estado do Espírito Santo. A inovação deste trabalho é a incorporação de uma análise de risco sobre a verdadeira disposição a pagar e, conseqüentemente, sobre o valor econômico total do PACM, o que significa maior rigor estatístico e maior confiabilidade na valoração econômica, tornando assim a avaliação mais condizente com a realidade.

\subsection{Método de valoração contingente - MVC}

O método de valoração contingente busca, por meio de surveys (entrevistas) pessoais, revelar as preferências dos indivíduos por um bem ou serviço ambiental; conseqüentemente, busca captar a disposição a pagar (DAP) para garantir um benefício ou a disposição a aceitar (DAC) para incorrer em um malefício.

Conforme FARIA e NOGUEIRA (1998), a idéia central do MVC é que indivíduos possuem diferentes graus de preferência por um bem ou serviço ambiental. Essa preferência torna-se visível quando os consumidores vão ao "mercado" e pagam valores por tais ativos.

O MVC estima uma medida monetária extraída de entrevistas que tendem a refletir as preferências expressas por consumidores, relativas ao acréscimo/decréscimo na qualidade de ativos ambientais.

O enfoque utilizado neste estudo, para determinar o valor econômico total do Parque “Chico Mendes”, é o de HANEMANN (1984); conforme BELLUZZO Jr. (1995), sua abordagem foi completada por SELLAR et al. (1985) e SELLAR et al. (1986), em que as características da demanda hicksiana (invertida) implicadas pelas formas funcionais utilizadas são analisadas. 


\section{Referencial teórico}

A teoria do consumidor postula que os indivíduos são racionais e se comportam para atingir o maior nível possível de satisfação. Logo, se as preferências são ordenadas, elas podem ser representadas pela função de utilidade direta (FUD), da seguinte forma:

$$
\mathrm{U}=\mathrm{U}(\mathrm{X}, \mathrm{Q}, \mathrm{T})
$$

em que, X é um vetor de quantidade dos bens de mercado; Q, vetor de bens públicos (recursos e serviços ambientais), em que as quantidades são fixadas pelo indivíduo; e T, vetor de tempo utilizado em várias atividades que produzem a utilidade do indivíduo.

Considerando-se que as escolhas dos indivíduos objetivam maximizar sua utilidade, dados os preços e dada a existência de uma restrição orçamentária, sendo a renda fixa, o problema de maximização de utilidade do consumidor pode ser expresso da seguinte forma:

$$
\begin{aligned}
& \text { Max } \mathrm{U}=\mathrm{U}(\mathrm{X}), \\
& \text { s.a } \sum_{i} P_{i} \times X_{i}=M,
\end{aligned}
$$

em que, $\mathrm{X}$ é um vetor de quantidades $\left(\mathrm{X}=\mathrm{X}_{1}, \mathrm{X}_{2}, \mathrm{X}_{3} \ldots, \mathrm{X}_{\mathrm{n}}\right)$; $P$, vetor de preços $\left(\mathrm{P}=\mathrm{P}_{1}, \mathrm{P}_{2}, \mathrm{P}_{3}, \ldots, \mathrm{P}_{\mathrm{n}}\right)$; e $M$, renda.

A solução desse problema fornece a função de demanda ordinária (função de demanda marshaliana), dada por:

$$
X_{i}=X_{i}(P, M)
$$

A equação (3) relaciona a quantidade ótima de determinado bem com os preços e com a renda monetária constante. Ao determinar as quantidades ótimas de cada bem $\mathrm{X}_{\mathrm{i}}$, o consumidor estará atingindo o maior nível possível de utilidade. A partir da determinação das demandas marshalianas é possível quantificar o excedente do consumidor. Contudo, conforme salientou BELLUZZO Jr. (1995), somente pela utilização 
de suposições não muito realistas de que as curvas de demanda sejam homotéticas ou quase lineares é que se pode garantir que o excedente do consumidor quantifique com exatidão, o bem-estar do consumidor.

Em face dessas deficiências, HICKS (1940/1 - 1945/6) formulou os conceitos de variação compensatória (VC) e variação equivalente (VE) ${ }^{3}$.

Pode-se entender VC como o montante pelo qual se aumenta (ou diminui) a renda do consumidor, para que, após uma modificação dos preços, ele esteja tão bem quanto na situação inicial, ou seja, para que ele permaneça na mesma curva de indiferença, representada por:

$$
v\left(P_{1}, M-Z_{1} q_{1}^{0}, q_{1}^{0}\right)=v\left(P_{1}, M-z_{1} q_{1}^{1}-V C, q_{1}^{1}\right),
$$

em que, $\mathrm{M}$ é a renda do consumidor; $\mathrm{Z}_{1} \mathrm{q}_{1}{ }^{0}$, parcela da renda gasta com o referido bem ambiental.

A primeira análise, referente à expressão (4), consiste em verificar o lado esquerdo da equação, que representa o máximo nível de utilidade obtido pelo indivíduo, dado pela quantidade $\mathrm{q}_{1}^{0}$. Já o lado direito representa o máximo nível de utilidade obtido pelo consumidor, dado pelo decréscimo na quantidade do bem que disponibilizou um valor superior, dado por $\mathrm{q}_{1}{ }^{1}$. Consequentemente, o VC é o valor da renda que o individuo deve compensar pelo aumento de $\mathrm{q}_{1}{ }^{0}$ para $\mathrm{q}_{1}{ }^{1}$, de forma a permanecer no nível de utilidade inicial, ou seja, estabelecer a igualdade entre os termos da equação acima.

Dessa forma, conforme salientou FREEMAN III (1993), a variação compensatória corresponde à seguinte pergunta:

- Você estaria disposto a pagar R\$ X para que a disponibilidade de serviços ambientais fornecidos pelo Parque Ambiental "Chico Mendes" não diminua? Ou seja, a VC é quanto o indivíduo está disposto a pagar para manter seu nível de satisfação.

\footnotetext{
${ }^{3}$ A variação equivalente (VE) corresponde ao montante da variação da renda que possui o mesmo efeito sobre o nível da utilidade que uma mudança de preço. É válido ressaltar que este montante irá conduzi-lo ao nível superior de utilidade. O conceito de VE não será tratado neste artigo.
} 


\section{Referencial analítico}

\subsection{Modelos econométricos}

Dois modelos econométricos foram utilizados para atingir os objetivos deste estudo. O primeiro é dado por:

$$
Y=X \beta+e,
$$

em que, Y corresponde a um vetor contendo as disposições a pagar manifestadas (maior lance aceito) em $\mathrm{R} \$$; $X$ representa um vetor de variáveis exógenas (renda familiar, sexo, anos de estudos e os vieses associados); $\beta$ representa um vetor de parâmetros desconhecidos a serem estimados pelo método dos mínimos quadrados ordinários; e e é o erro aleatório com as pressuposições usuais.

O segundo é o modelo logit, que possibilita a determinação da verdadeira disposição a pagar (true stated willingness-to-pay). Metodologia semelhante foi utilizada por HADKER et. al. (1997), em um estudo sobre a disposição a pagar do Borivli National Park, em Bombay - Índia. No modelo logit, estima-se, primeiro, a probabilidade de um indivíduo se dispor a pagar e, em seguida, o preço, em que a probabilidade de obtenção de uma resposta SIM seja de $50 \%$, considerada o valor máximo que um indivíduo estaria disposto a pagar.

Esse modelo está baseado na função de probabilidade logística acumulada, representada da seguinte forma:

$$
P_{i}=F\left(X_{i} \beta\right)=\frac{1}{1+e^{-X_{i} \beta}},
$$

em que X é um vetor de variáveis explicativas; e $\beta$, parâmetros desconhecidos a serem estimados; e $\mathrm{P}_{\mathrm{i}}$ probabilidade de o indivíduo i responder SIM à indagação se está disposto a pagar para usufruir dos benefícios resultantes da conservação e manutenção de uma área ambiental.

A variável $P_{i}$ não é observada. Observa-se $Y_{i}=1$, quando o indivíduo responder SIM e $Y_{i}=0$, quando o a resposta for NÃO. Dessa forma, a estimação dos parâmetros ( $\beta$ ), em (6), deve ser feita por Máxima Verossimilhança. (GREENE, 1997). 


\subsection{Estimativas dos benefícios}

Como um dos objetivos do trabalho é estimar o valor econômico total do PACM, formulou-se a seguinte pergunta: Sabendo que o PACM é o único parque ambiental de Rio Branco, você estaria disposto a pagar R \$ P para possibilitar a manutenção e a conservação do parque para você e para seus familiares atuais e futuros?

Quando o entrevistado respondia SIM, era apresentado um lance superior ao anterior; caso respondesse $\mathrm{SIM}^{4}$ novamente, outro lance superior seria ofertado, e assim sucessivamente, até o indivíduo responder NÃO. Caso os lances ficassem abaixo da máxima DAP, seria considerado pago; caso, os lances ficassem acima da DAP máxima, não. Logo, serão consideradas as respostas afirmativas igual a 1 . Nos casos em que 0 indivíduo respondesse NÃO, seria oferecido um valor inferior e assim sucessivamente, até determinar um valor que ela aceitasse pagar. Nesse caso, considerou-se a resposta negativa igual a 0 .

Supondo que o entrevistado derive sua função de utilidade da conservação e manutenção do PACM e de sua renda, pode-se representá-la da seguinte forma:

$$
\mathrm{U}=\mathrm{U}(\mathrm{J}, \mathrm{Y}, \mathrm{S}) \text {, }
$$

em que $\mathrm{J}$ é uma variável binária; $\mathrm{J}=1$ significa contribuir para manutenção e conservação do PACM; J = 0, não contribui; Y, renda; S vetor de outros atributos que podem influenciar a DAP.

Conseqüentemente, pode-se representar duas funções de utilidade para o indivíduo, dadas por:

$$
\begin{aligned}
& \mathrm{U}_{0}=\mathrm{U}(0, \mathrm{Y}, \mathrm{S}), \\
& \mathrm{U}_{1}=\mathrm{U}(1, \mathrm{Y}, \mathrm{S}) .
\end{aligned}
$$

\footnotetext{
${ }^{4} \mathrm{O}$ procedimento utilizado para negociar os lances com os entrevistados foi o referendum com o bidding games, em que vários valores foram negociados com o intuito de captar a verdadeira disposição a pagar. Pela técnica Dephi foram determinados onze valores, que foram negociados nas entrevistas. $O$ primeiro lance correspondia a um lance médio ou mediano.
} 
A equação (8) indica que o indivíduo não aceita contribuir, enquanto (9), o contrário.

Conforme AGUIRRE e FARIA (1996), $\mathrm{U}_{0}$ e $\mathrm{U}_{1}$ são variáveis aleatórias com distribuição de probabilidade e com médias v(0,Y,S) e v(1,Y,S). Logo, as utilidades podem ser escritas da seguinte forma:

$$
\mathrm{U}(\mathrm{J}, \mathrm{Y}, \mathrm{S})=\mathrm{v}(\mathrm{J}, \mathrm{Y}, \mathrm{S})+\varepsilon_{\mathrm{j}} \mathrm{J}=0,1
$$

em que $\varepsilon_{0}$ e $\varepsilon_{1}$ são variáveis independentes e identicamente distribuídas, com média zero e variância finita.

O entrevistado responderá SIM à pergunta acima, apenas se:

$$
U_{1}(1, Y-D A P, S)-v(0, Y, S) \geq U_{0}(0, Y, S)
$$

Do ponto de vista estatístico, a resposta do indivíduo é uma variável aleatória, em que a distribuição de probabilidade (Pr), é dada por:

$$
\begin{aligned}
& P_{1}=\operatorname{Pr}\{\text { Aceita contribuir }\} \\
& P_{1}=\operatorname{Pr}\left\{v\left(1, Y-D A P, S+\varepsilon_{1} \geq v(0, Y, S)+\varepsilon_{0}\right\}\right. \\
& P_{1}=\operatorname{Pr}\{\Delta v \geq \delta\}
\end{aligned}
$$

em que

$$
\begin{aligned}
& \Delta v=v(1, Y-D A P, S)-v(0, Y, S) \\
& \delta=\varepsilon_{0}-\varepsilon_{1},
\end{aligned}
$$

por fim,

$$
\begin{aligned}
& P_{0}=\operatorname{Pr}\{\text { não contribui } \\
& P_{0}=1-\operatorname{Pr} .
\end{aligned}
$$

Representando a distribuição de probabilidade por $\mathrm{F}(\bullet)$, a probabilidade de um entrevistado responder afirmativamente é dada por

$$
P_{1}=F_{\delta}(\Delta v) .
$$


Ao optar pela distribuição logística, tem-se:

$$
P_{1}=F_{\delta}(\Delta v)=\left(1+e^{-\Delta v}\right)^{-1}
$$

O modelo logit, conforme AGUIRRE e FARIA (1996), será interpretado como resultado de uma escolha que maximiza a utilidade. A função $\mathrm{F}_{\delta}(\bullet)$ deve ter a forma (16), condição que é análoga às condições da teoria da demanda.

Calculando $\mathrm{v}$, pode-se determinar o modelo estatístico discreto de escolha, supondo que:

$$
\begin{aligned}
& v(J, Y, S)=a_{j}(S)+b Y, \mathrm{~J}=0,1 \\
& \text { em que b > 0. Logo, } \\
& \begin{aligned}
\Delta v & =a_{1}(S)+b(Y-D A P)-a_{0}(S)-b y \\
\Delta v & =\left[a_{1}(S)-a_{0}(S)-b D A P\right] \\
\Delta v & \left.=\left(a_{1}-a_{0}\right)-b D A P\right) \\
\Delta v & =a-b D A P
\end{aligned}
\end{aligned}
$$

Com base na equação acima, o modelo estatístico discreto de escolha será dado por

$$
\begin{aligned}
& P_{1}=F_{\delta}(a-b D A P), \\
& \text { em que } \mathrm{a}=\mathrm{a}_{1}-\mathrm{a}_{0} .
\end{aligned}
$$

O objetivo do MVC é calcular uma medida baseada na utilidade do valor atribuído à disponibilidade do ativo ambiental, usando-se um modelo de escolha binária, ou seja, trata-se de estimar uma DAP que satisfaça à seguinte igualdade:

$$
U\left(1, Y-D A P^{*}, S\right)=U(0, Y, S)
$$

de outra forma,

$$
v\left(1, Y-D A P^{*}, S\right)-v(0, Y, S)=\delta
$$


Conforme AGUIRRE e FARIA (1996), $\delta$ possui distribuição logística padronizada, e a mediana é igual à média e igual a zero. Logo, $\delta=0$ está associado ao ponto de indiferença, sendo $\mathrm{F}_{\delta}=0,5$. Para $\Delta(\mathrm{v})=\delta=0$, o entrevistado está indiferente entre aceitar/rejeitar a implantação do projeto, e o valor médio (mediano) da DAP é considerado como o valor que o indivíduo está disposto a pagar por DAP*. Logo, pode-se escrever:

$\operatorname{Pr}(\Delta \mathrm{v}=\delta=0)=\mathrm{F}_{\delta}(\Delta \mathrm{v}=0)=0,5$

DAP* satisfaz à seguinte condição:

$\mathrm{v}\left(\mathrm{DAP}^{*}\right)=0$,

ou, considerando-se (17):

$\mathrm{a}-\mathrm{bDAP}^{*}=0$

que implica:

$D A P^{*}=\frac{a}{b}$

A equação (21) representa a DAP, ou seja, indica que o valor médio (mediano) é calculado em função dos coeficientes do modelo estatístico discreto de escolha binária. No caso em que o modelo apresentar mais de uma variável independente, o denominador será o parâmetro da variável preço e o numerador, o intercepto mais os valores médios das demais variáveis, multiplicado pelos seus respectivos parâmetros.

\subsection{Determinação do intervalo de confiança para DAP}

A determinação de um intervalo de confiança para DAP é importante para uma avaliação de sua precisão estatística e possibilita uma avaliação mais criteriosa por parte dos tomadores de decisão.

$\mathrm{O}$ bootstrapping consiste em estimar o modelo usual e gerar $\mathrm{T}$ amostras aleatórias, com reposição dos elementos do vetor do resíduo, dado por $e=y-X \hat{\beta}$. Utilizando-se os vetores $\mathrm{T}$ de resíduos obtidos, $\mathrm{e}^{*}$ gera-se 
um novo, regressando de acordo com $Y^{*}=X \hat{\beta}+e^{*}$. Por último, para cada um dos conjuntos de variáveis dependentes gerados no modelo são re-estimados, logo, serão obtidos T vetores de parâmetro $\hat{\beta} *$, que serão utilizados na construção da distribuição empírica da DAP.

\subsection{Método de Monte Carlo}

O método de Monte Carlo é uma tradicional técnica de amostragem de número aleatório ou pseudo-aleatório de uma distribuição de probabilidade. O processo de amostragem de Monte Carlos é completamente aleatório, o que significa dizer que qualquer elemento da amostragem pode provir de determinada faixa da distribuição de probabilidade. Naturalmente, a amostragem é mais provável em área onde exista maior probabilidade de ocorrência. Cada amostragem de Monte Carlo irá utilizar um número aleatório entre zero e um. Com muitas interações, a amostragem de Monte Carlo irá recriar uma distribuição de probabilidade por meio de amostragem. Esse processo de simulação possibilitará uma análise de risco sobre a verdadeira disposição a pagar.

\subsection{Fonte de dados}

Os dados utilizados neste trabalho foram procedentes de fonte primária, coletada mediante entrevista no PACM. Determinou-se uma amostra de 256 indivíduos $^{5}$, no entanto, adicionaram-se $10 \%$ de margem de segurança, ficando assim com 282 entrevistas. A aplicação dos questionários deu-se nos dias de sábado e domingo, no período de 29 de junho a 21 de julho de 2002, totalizando quatro semanas. Aos domingos, maior número de questionários eram aplicados, em torno de 45, contra uma média de 20, nos sábados.

\section{Resultados e discussões}

Do total da amostra, 68,80\% proviam do $1 .{ }^{\circ}$ distrito de Rio Branco ${ }^{6}$;

\footnotetext{
${ }^{5} \mathrm{O}$ tamanho da amostra foi determinado com base em métodos estatísticos de dimensionamento da amostra, especificamente, amostra infinita.

${ }^{6}$ Historicamente, Rio Branco é dividida em dois distritos, que são separados pelo rio Acre.
} 
$29 \%$, do $2 .^{\circ}$ distrito; e apenas $2,2 \%^{7}$, de outros locais (zona urbana, municípios vizinhos, etc.). A primeira, segunda, terceira, quarta e quinta regiões apresentaram as seguintes taxas de visitação, 29,03\% , 13,98\%, $19,71 \%, 27,60 \%$ e 9,68\%. Esses resultados eram esperados, no entanto, o que surpreendeu foi a taxa de visitação da quarta região, em virtude de esta ser bastante afastada do parque.

A disposição a pagar manifestada versus o nível de renda dos visitantes indica que no segundo estrato concentrava a maior proporção de contribuintes, nesta classe, $77,27 \%$ dos entrevistados se dispunha a contribuir com o PACM. Este comportamento é seguido, em menor escala, pelo terceiro e pelo primeiro nível de renda, com, respectivamente, $70 \%$ e $67,10 \%$; os demais níveis possuíam taxas inferiores a 62,5\%, o que possibilita maiores inferências acerca dessa situação (Tabela 1).

Tabela 1 - Níveis de renda dos visitantes do PACM versus DAP manifestada - PACM, $2002^{8}$

\begin{tabular}{|c|c|c|c|c|c|c|c|c|c|c|c|c|}
\hline \multirow[b]{2}{*}{$\begin{array}{c}\text { Níveis } \\
\text { de renda }\end{array}$} & \multicolumn{11}{|c|}{ DAP } & \multirow[b]{2}{*}{ Total } \\
\hline & 0,00 & 1,00 & 2,50 & 4,00 & 5,50 & 7,50 & 10,50 & 12,00 & 14,50 & 19,00 & 25,00 & \\
\hline $0,00-\quad 600,00$ & 50 & 15 & 23 & 16 & 23 & 14 & 5 & 4 & - & 2 & - & 152 \\
\hline $601,00-1.200,00$ & 15 & 4 & 6 & 9 & 11 & 8 & 5 & 7 & - & 1 & - & 66 \\
\hline $1.201,00-1.800,00$ & 6 & - & 1 & 3 & 3 & 1 & 2 & 2 & 2 & - & - & 20 \\
\hline $1.801,00-2.400,00$ & 6 & 1 & - & 1 & 3 & - & 1 & 1 & 1 & 1 & 1 & 16 \\
\hline $2.401,00-3.000,00$ & - & - & - & - & - & - & - & - & - & - & - & - \\
\hline $3.001,00-3.600,00$ & 6 & - & 1 & - & 2 & - & 3 & - & 1 & 1 & 2 & 16 \\
\hline $3.601,00-4.200,00$ & 1 & - & - & - & - & - & - & - & - & - & - & 1 \\
\hline $4.201,00-4.800,00$ & 3 & - & - & - & - & - & - & 2 & - & - & - & 5 \\
\hline $5.401,00-6.000,00$ & 1 & - & - & - & - & - & - & - & - & - & - & 1 \\
\hline$>6.000,00$ & 1 & - & - & - & - & - & - & - & - & - & 1 & 2 \\
\hline Total & 89 & 20 & 31 & 29 & 42 & 23 & 16 & 16 & 4 & 5 & 4 & 279 \\
\hline
\end{tabular}

Fonte: Resultados da pesquisa.

A Prefeitura Municipal de Rio Branco - PMRB utiliza para a elaboração do orçamento participativo uma divisão destes distritos em regiões. No $1^{\circ}$ distrito de Rio Branco, estão situadas a $2^{a}, 3^{a}, 4^{a}$ e $5^{a}$ regiões, enquanto, no $2^{\circ}$ distrito está situada apenas a 1. ${ }^{a}$ região administrativa.

${ }^{7}$ Em todas as tabulações estes entrevistados foram agregados no $1^{\circ}$ distrito na $5^{\mathrm{a}}$ região.

${ }^{8}$ Os valores da DAP foram determinados pela aplicação de 11 questionários a especialistas (técnica Delphi) que a determinaram pelos níveis de renda constante nesta Tabela. 
Valoração Contingente do Parque "Chico Mendes": uma Aplicação Probabilística do Método Referendum com Bidding Games

A disposição a pagar manifestada versus o nível de escolaridade indica que os entrevistados que tinham o segundo grau apresentavam a maior propensão a contribuir para a manutenção e conservação do PACM, pois, aproximadamente, $75 \%$ dos entrevistados com este nível de escolaridade se dispuseram a contribuir. Os entrevistados com nível superior $61,4 \%$ se dispunham a contribuir. Em relação aos entrevistados sem instrução ou apenas com o ensino fundamental, constatou-se que $60 \%$ e $67 \%$ dispunham-se a contribuir.

Observa-se que $32 \%$ dos entrevistados não se dispuseram a contribuir com nenhum valor para manutenção e preservação do PACM. Diversos motivos explicam o não pagamento da contribuição, dentre eles, os vieses associados ao método e a variáveis econômicas (desemprego ou renda familiar inferior a $\mathrm{R} \$ 200,00)$. A Tabela 2 faz uma descrição dos motivos associados aos lances nulos.

Tabela 2 - Vieses associados às DAPs nulas do PACM, $2002^{9}$

\begin{tabular}{lcccc}
\hline \multicolumn{1}{c}{ Vieses } & Freqüência & $\%$ & $\%$ válida & \% cumulativa \\
\hline Motivos econômicos & 15 & 5,40 & 16,85 & 16,85 \\
Viés do veículo de pagamento & 11 & 3,90 & 12,38 & 29,23 \\
Viés de protesto & 20 & 7,17 & 22,47 & 51,70 \\
É uma função da prefeitura & 43 & 15,43 & 48,30 & 100,00 \\
Total dos vieses & 89 & 31,90 & 100,00 & - \\
Total dos não-vieses & 190 & 68,10 & - & - \\
Total geral & 279 & 100,00 & - & - \\
\hline
\end{tabular}

Fonte: Resultados da pesquisa.

Na Tabela 2, verifica-se que os motivos econômicos ${ }^{10}$ destacaram-se como um dos fatores explicativos da DAP nula. É importante destacar que os lances não-nulos não significam a não presença de viés ${ }^{11}$.

\footnotetext{
${ }^{9}$ Dois questionários foram excluídos.

10 Nos estudos conduzidos no exterior a presença de uma DAP nula em decorrência de motivos econômicos é praticamente inexistente, entretanto, levando-se em conta que esta pesquisa foi conduzida em um estado pobre, em que existe um alto índice de desemprego, uma DAP que reflita diretamente uma medida monetária pode trazer problemas ao método. No entanto, uma forma de contornar este problema seria perguntar ao indivíduo se ele se disporia a contribuir com o parque em forma de serviços prestados e posteriormente, transformar estas horas de serviços prestados em um valor monetário, este valor seria a DAP do indivíduo, em outras palavras, seria uma proxy.
} 
O viés de veículo de pagamento mostrou-se presente. A justificativa dos entrevistados para não aceitarem os lances oferecidos foi que seria mais apropriada a cobrança de uma taxa na entrada do parque.

$\mathrm{O}$ viés de protesto agrupa uma série de justificativas contidas nos questionários, quais sejam: a) Não acredita que, ao pagar, o parque terá melhor manutenção e será conservado; b) Não acredita que necessite pagar uma taxa para que o parque seja conservado e tenha melhor manutenção; c) Já paga muitos impostos; e d) Outros motivos.

Apesar de os vieses de protesto terem sido agregados, tanto em termos absolutos quanto em termos relativos, a maior justificativa para uma DAP nula foi: é uma função da prefeitura.

Nesse momento, o indivíduo tira de si qualquer responsabilidade individual sobre a utilização de um ativo ambiental e deixa toda a responsabilidade para o poder público. O problema, nesse tipo de concepção, reside no fato de essa postura incentivar a sociedade a ficar fora do processo de tomada de decisão no gerenciamento dos recursos naturais. O gerenciamento desse tipo de recursos influencia, diretamente, a qualidade de vida da sociedade presente e das futuras gerações.

A DAP máxima manifestada pelo entrevistado, ou seja, o maior lance que ele aceitou pagar foi estimado pelo MQO, com correção da heterocedasticidade pelo método de White. Conforme Tabela 3 o coeficiente de correlação - $\mathrm{R}^{2}$ foi apenas razoável, no entanto, esse resultado é semelhante a resultados encontrados em pesquisas que utilizaram a mesma metodologia, tanto em nível nacional como internacional. Em parte, isso se justifica por não haver um padrão que determine quanto será o lance máximo do indivíduo.

\footnotetext{
${ }^{11}$ No entanto, a captação dos vieses associados ao aceite de pagamento não é detectada estatisticamente. Conseqüentemente, fica na responsabilidade do entrevistador detectar vieses e descartar os questionários com este problema.
} 
Valoração Contingente do Parque "Chico Mendes": uma Aplicação Probabilística do Método Referendum com Bidding Games

Tabela 3 - Resultados econométricos da stated willingness - to-pay para o PACM, 2002

\begin{tabular}{cccccc}
\hline Variável & Coeficiente & Erro-padrão & T-Student & Valor-P & Elasticidades \\
\hline C & $2,815843^{* * *}$ & 0,605103 & 4,653496 & 0,000000 & - \\
RF & $0,000714^{* * *}$ & 0,000125 & 5,694920 & 0,000000 & 0,2276 \\
SX & $1,462570^{* * *}$ & 0,442985 & 3,301622 & 0,001100 & - \\
AEST & $0,142145^{* *}$ & 0,060424 & 2,352475 & 0,019400 & 0,3020 \\
DVD1 & $-6,465553^{* * *}$ & 0,437421 & $-14,78108$ & 0,000000 & - \\
DVD2 & $-6,916805^{* * *}$ & 0,527750 & $-13,10622$ & 0,000000 & - \\
DVD3 & $-6,756903^{* * *}$ & 0,818516 & $-8,255062$ & 0,000000 & - \\
DVD4 & $-5,705190^{* * *}$ & 0,589655 & $-9,675477$ & 0,000000 & - \\
\hline$R^{2}$ & 0,436877 & F-statistic & 30,03496 & & \\
$\mathrm{R}^{2}$ ajustado & 0,422331 & P-valor & 0,000000 & & \\
DAP Média & 4,410000 & Variância & 5,03 & & \\
\hline
\end{tabular}

Fonte: Resultados da pesquisa.

$* * *$ significativo a $1 \% ; * *$ significativo a $5 \%$.

DAP é variável dependente e corresponde a disposição máxima a pagar do indivíduo; RF é a renda familiar mensal em reais; AEST é número de anos de estudo formal feito pelo entrevistado; SX é uma variável dummy: 1 - homem; 0 - mulher; DVD1 dummy de não pagamento por motivos econômicos; DVD2 dummy de não pagamento - viés do veículo de pagamento; DVD3 dummy de não pagamento - viés de protesto (geral); e DVD4 dummy de não pagamento - viés de protesto (é uma função da prefeitura). Aceita a contribuir é a categoria base dos vieses.

Número de observações: 279

O efeito marginal da renda demonstra que uma variação da renda de $\mathrm{R}$ \$ 1.000,00 acarretará uma variação de $\mathrm{R}$ \$ 0,71 sobre a DAP; em relação à instrução, uma variação em um ano de estudo ocasionará uma variação de R\$ 0,14 sobre a DAP. O efeito marginal das dummies utilizadas para captarem os motivos de não pagamento informa que, em relação a DVD1, o surgimento deste viés ocasionará diminuição da DAP de, aproximadamente, R\$3,65; DVD2 indica que o viés de veículo de pagamento acarretará diminuição de R 4,10 ; DVD3, a presença do viés de protesto, de forma geral, diminui a DAP em $\mathrm{R} \$ 3,95$, por último, o viés de protesto específico: "é uma função da prefeitura”, acarreta uma diminuição da DAP da ordem de $\mathrm{R} \$ 2,90$. Como pode ser observado, o impacto dos vieses e dos motivos econômicos na DAP teve grande magnitude.

Todos os vieses e o motivo econômico possuem o sinal esperado, pois seu impacto influencia, de forma negativa, a disposição a pagar dos indivíduos.

O modelo logit foi utilizado na estimação da verdadeira disposição a pagar (VDAP). A operacionalização da modelagem seguiu a mesma me- 
todologia descrita em BELLUZZO JR. (1999). Para construção do modelo foram descartados os questionários que apresentaram algum tipo de viés, que corresponderam a $26,5 \%$ da amostra, ou alegaram motivos econômicos para a não contribuição, que totalizam a 5,40\% da amostra.

Tabela 4 - Parâmetros estimados do modelo logit para a verdadeira disposição a pagar para manutenção e conservação, do PACM, 2002

\begin{tabular}{ccccccc}
\hline Variável & Coeficiente & Erro-padrão & t-student & P-valor & Elasticidades & $\begin{array}{c}\text { Efeito } \\
\text { marginal }\end{array}$ \\
\hline C & $0,978228^{* * *}$ & 0,351656 & 2,781774 & 0,0054 & - & - \\
DAP & $-0,370504^{* * *}$ & 0,018967 & $-19,53384$ & 0,0000 & $-3,00273$ & $-6,781400$ \\
RF & $0,000503^{* * *}$ & $8,19 \mathrm{E}-05$ & 6,145635 & 0,0000 & 0,536178 & 0,009200 \\
SX & $0,706094^{* * *}$ & 0,139484 & 5,062177 & 0,0000 & 0,358121 & 0,129238 \\
ID & $0,0128850^{*}$ & 0,007547 & 1,707381 & 0,0878 & 0,338127 & 0,235800 \\
AEST & $0,022431^{\text {NS }}$ & 0,021670 & 1,035097 & 0,3006 & 0,158467 & 0,410600 \\
Log likelihood & $-751,375$ & & & & & \\
Restr. Log likelihood & $-1.393,994$ & & & & & \\
LR statistics & $1.285,238$ & & & & & \\
Probability & 0,000 & & & & & \\
\hline
\end{tabular}

Fonte: Resultados da pesquisa.

*** significativo a $1 \% ;{ }^{* *}$ significativo a $5 \%$; * significativo a $10 \%$; e NS não-significativo.

$\mathrm{v}$ é a variável dependente - Probabilidade do indivíduo se dispor a pagar; ID corresponde à idade do entrevistado; Amostra $1-2.090$.

Os sinais apresentados no modelo estimado expressam sua conformidade com a teoria econômica. Excluindo o parâmetro AEST, os demais foram significativos a 1 e $10 \%$, em relação a medidas de qualidade de ajustamento; o Índice de Razão de Verossimilhança (LRI) indica que 46,09\% das variações nas variáveis independentes explicam as variações da variável dicotômica. ${ }^{13}$ O Teste de razão de Verossimilhança (LR) ${ }^{14}$

\footnotetext{
${ }^{12}$ Para a operacionalização deste modelo seguiu-se a seguinte metodologia. Oferecia-se um valor aleatório da DAP para o entrevistado, caso aceita-se, valores superiores eram oferecidos até atingir um valor máximo. Quando o entrevistado não aceitava o valor inicial, valores inferiores eram oferecidos. Logo, cada lance pode ser representado como valores dicotômicos (aceita, sim, 1), (rejeita, não, 0). Por fim, o número total da amostra é dado por 11 (total dos lances oferecidos) multiplicado por (190) número de questionários válidos.

${ }^{13}$ Esta medida é popularmente conhecida como $\mathrm{R}^{2}$ de McFadden. É importante destacar que esta medida de qualidade de ajustamento deve ser observada com ressalvas, pois não é objetivo do modelo Logit estimar uma reta sobre valores dicotômicos.

${ }^{14} \mathrm{~A}$ interpretação deste teste é semelhante ao do teste $\mathrm{F}$.
} 
indica que pelo menos um parâmetro do modelo ajustado é diferente de zero. Por fim, o modelo apresentou alto poder de predição, pois cerca $85 \%$ de suas predicações são corretas.

A probabilidade média de o indivíduo aceitar contribuir com a manutenção e conservação do PACM é de 24,12\%. Mantidas as demais variáveis constantes, a dummy de sexo - SX indica um acréscimo de, aproximadamente, 13 pontos percentuais sobre a probabilidade de ocorrência do evento, quando o entrevistado é do sexo masculino. Esse resultado ocorre em virtude de o homem contribuir com maior parcela na renda familiar, conseqüentemente, possui maior peso nas decisões que envolvem dispêndios no orçamento.

O efeito marginal de ID mostra que a variação de um ano de idade do indivíduo acarreta uma variação de 0,2358 pontos percentuais na probabilidade de o indivíduo aceitar a contribuir. Em parte, esse resultado pode indicar que, com o passar do tempo, o surgimento de programas, políticas e campanhas educativas influenciem a VDAP do indivíduo.

Em relação à renda familiar - RF, verificou-se que o efeito marginal dessa variável sobre a probabilidade de o indivíduo pagar é pequena, pois uma variação na renda familiar do indivíduo em uma unidade monetária acarreta um impacto de 0,0092 pontos percentuais.

O impacto do valor da VDAP na probabilidade de o individuo aceitar a contribuir, conforme esperado, é negativo; indica que há um relacionamento inverso entre as variáveis, ou seja, à medida que o valor da VDAP aumenta, diminui a probabilidade de o individuo aceitar a pagar. Em outras palavras, um aumento da VDAP em uma unidade monetária acarretará diminuição da probabilidade de pagamento em 6,78 pontos percentuais, o que representa uma relação de demanda.

Por último, sobre a variável AEST (anos de estudo) não foi possível fazer inferências, pois a probabilidade de seu parâmetro ser igual a zero é alta.

As elasticidades de probabilidade indicam que uma variação de $10 \%$ na verdadeira DAP ocasiona uma variação inversa de 30,02 \% na probabilidade do indivíduo se dispor a contribuir, ou seja, uma relação elástica; uma variação de $10 \%$ na renda familiar ocasiona uma variação direta de 5,36\% na probabilidade de ocorrência do evento, o que caracteriza o PACM como sendo um "bem normal”; uma variação de $10 \%$ na idade 
do indivíduo ocasiona uma variação direta de $1,58 \%$ na probabilidade do indivíduo contribuir.

Teoricamente, espera-se que, quanto maior for o lance, menor será a probabilidade de ocorrência de o indivíduo aceitar a contribuir, fato presente neste estudo. Verificou-se que o lance de $\mathrm{R} \$ 1,00$ possui uma probabilidade estimada de ocorrência de $90 \%$, enquanto o de $\mathrm{R} \$ 25,00$, apenas de $1 \%$. (Tabela 5).

Tabela 5 - Probabilidades observadas e calculadas de ocorrência da VDAP no PACM, 2002

\begin{tabular}{lllllllllllll}
\hline \multicolumn{1}{c}{ Lances oferecidos } \\
Especificação & 1,00 & 2,50 & 4,00 & 5,50 & 7,5 & 10,50 & 12,00 & 14,50 & 16,00 & 19,00 & 25,00 \\
\hline $\begin{array}{c}\text { Probabilidade } \\
\text { de ocorrência da } \\
\text { VDAP }\end{array}$ & $90 \%$ & $84 \%$ & $76 \%$ & $65 \%$ & $48 \%$ & $26 \%$ & $18 \%$ & $9 \%$ & $6 \%$ & $2 \%$ & $1 \%$ \\
& & & & & & & & & & \\
VDAP observada & $100 \%$ & $90 \%$ & $74 \%$ & $60 \%$ & $37 \%$ & $25 \%$ & $16 \%$ & $8 \%$ & $6 \%$ & $6 \%$ & $4 \%$ \\
\hline
\end{tabular}

Fonte: Resultado da pesquisa

Conforme Tabela 5, a probabilidade de aceite do lance varia de acordo com o valor apresentado da VDAP. Observa-se que a passagem de $\mathrm{R} \$ 5,50$ para $\mathrm{R}$ \$ 7,50 diminui em 17 pontos percentuais a probabilidade de o indivíduo se dispor a pagar. A magnitude desse evento é superada quando a VDAP sofre um acréscimo de $R \$ 7,50$ para $R \$ 10,50$, pois esta diminui em 22 pontos percentuais. A partir de $\mathrm{R} \$ 10,50$, o impacto do acréscimo da VDAP sobre a probabilidade do indivíduo responder "SIM" diminuiu sensivelmente.

A verdadeira disposição a pagar para manutenção e conservação foi estimada em R $\$ 7,60$ ou, aproximadamente, US $\$ 2,60$. Este valor indica uma proxy do preço que o indivíduo estar disposto a pagar mensalmente pela conservação e manutenção do PACM, ou de outra maneira, é um indicativo das preferências dos consumidores por este ativo.

No entanto, esse valor não possui um intervalo de confiança que permita efetuar inferências seguras sobre a verdadeira DAP. Como forma de sanar esse problema, foi efetuado um bootstrapping para criar o desvio-padrão e um intervalo de confiança para a VDAP. Após realização do bootstrapping, foi possível determinar a distribuição de probabilidade 
que a true willingness-to-pay possui; nesse caso, o teste de Jarque-Bera ${ }^{15}$ indica uma distribuição normal. A Tabela 6 mostra as principais estatísticas dessa simulação.

Tabela 6 - Estatísticas do bootstrapping ${ }^{16}$ na VDAP do PACM, 2002

\begin{tabular}{cc}
\hline Especificações & Valores em R\$ \\
\hline Média & 7,885638 \\
Mediana & 7,886151 \\
Máximo & 8,528472 \\
Mínimo & 7,206914 \\
Desvio-padrão & 0,187285 \\
Jarque-Bera & 0,688122 \\
Probabilidade & 0,708886 \\
\hline
\end{tabular}

Fonte: Resultados da pesquisa.

Utilizando-se a média determinada pelo bootstrapping, chega-se a um VET $^{17}$ de R $\$ 23.946 .380,00$ ou U\$ 8.200.815,00 ${ }^{18}$. Em estudos similares, RIBEIRO (1998) e BELLUZZO JR. (1995) estimaram os benefícios oriundos da despoluição do rio Meia Ponte e do Tietê, respectivamente, de R\$ 162.810.308,60 (US\$ 139 milhões) e R \$ 900 milhões (US\$ 980 milhões). O VET do PACM fornece uma sinalização para dar suporte às decisões de políticas públicas voltadas para o gerenciamento desse recurso.

De posse dos dados fornecidos pelo bootstrapping, efetuou-se uma análise de risco, com o objetivo de determinar a probabilidade de ocorrência de determinada DAP e, conseqüentemente, do VET. A simulação de Monte Carlo, com utilização de uma distribuição T-Normal (média, desvio-padrão, mínimo e máximo), com um milhão de interações, indicou os valores constantes da Tabela 7 .

\footnotetext{
${ }^{15}$ Ver GUJARATI (2000).

${ }^{16}$ Para a operacionalização do bootstrapping foi criado um programa no Eviews 4.0. Foram realizadas 5.000 interações para a determinação dos desvios-padrão associados à verdadeira DAP.

${ }^{17}$ O VET é calculado da seguinte forma: multiplica-se a DAP por 12 (meses do ano), posteriormente, multiplica-se pelo total da população afetada.

${ }^{18}$ Três cotações foram utilizadas para a conversão para o PACM, US\$ $1,00=\mathrm{R} \$ 2,92$; para o rio Meia Ponte, US $\$ 1,00=1,16$; e para o rio Tietê, US\$ $1,00=0,917$.
} 
Tabela 7 - Análise de risco: VET e VDAP para o PACM, 2002

\begin{tabular}{ccc}
\hline Probabilidade de ocorrência & VET & DAP \\
\hline $5 \%$ & $23.011 .580,00$ & 7,5778 \\
$10 \%$ & $23.217 .860,00$ & 7,6457 \\
$15 \%$ & $23.357 .120,00$ & 7,6916 \\
$20 \%$ & $23.467 .840,00$ & 7,7281 \\
$25 \%$ & $23.562 .840,00$ & 7,7593 \\
$30 \%$ & $23.648 .160,00$ & 7,7874 \\
$35 \%$ & $23.727 .220,00$ & 7,8135 \\
$40 \%$ & $23.802 .250,00$ & 7,8382 \\
$45 \%$ & $23.874 .830,00$ & 7,8621 \\
$50 \%$ & $23.946 .380,00$ & 7,8856 \\
$55 \%$ & $24.017 .700,00$ & 7,9091 \\
$60 \%$ & $24.090 .290,00$ & 7,9330 \\
$65 \%$ & $24.165 .300,00$ & 7,9577 \\
$70 \%$ & $24.244 .350,00$ & 7,9838 \\
$75 \%$ & $24.329 .640,00$ & 8,0118 \\
$80 \%$ & $24.424 .610,00$ & 8,0431 \\
$85 \%$ & $24.535 .260,00$ & 8,0796 \\
$90 \%$ & $24.674 .390,00$ & 8,1254 \\
$95 \%$ & $24.880 .330,00$ & 8,1932 \\
Mínimo & $21.886 .840,00$ & 7,2074 \\
Média & $23.946 .070,00$ & 7,8855 \\
Máximo & $25.894 .920,00$ & 8,5273 \\
Desvio-padrão & $567.054,10$ & 0,1867 \\
\hline
\end{tabular}

Fonte: Resultados da pesquisa.

Com base na simulação de Monte Carlo, podem-se determinar as probabilidades de ocorrência de determinada VDAP e, conseqüentemente, do VET. Com $100 \%$ de probabilidade, a simulação de Monte Carlos indica que o valor econômico total da PACM é inferior a $\mathrm{R} \$ 25.894 .920,00$ (US\$ 8.868.812,00) e superior a R\$21.886.840,00 (US\$ 7.495.493,00); o valor médio de R\$ 23.946.380,00 (US\$ 8.200.815,00); representa o ponto de indiferença entre os valores extremos. Como este é o valor com maior probabilidade de ocorrência, é mais recomendável à análise de implementação de políticas ou programas que interfiram nos benefícios oriundos do PACM. A importância da análise de risco reside no fato de possibilitar inferência probabilística acerca da ocorrência de determinada DAP e, conseqüentemente, determinado VET. Pela determinação deste 
tipo de intervalo de confiança, minimizam-se os problemas oriundos do simples valor de uma VDAP e um VET qualquer.

\section{Conclusões}

A disposição a pagar pela manutenção e conservação do parque ambiental "Chico Mendes" indica uma proxy do preço que o ativo representa. Logo, a monetização das preferências dentro das limitações existentes (educação, renda, consciência ambiental e possíveis vieses e outras) possibilita uma sinalização de mercado com relação ao uso deste ativo. Com isso, é possível verificar se a sociedade está consciente de que melhorias ou danos no PACM possam refletir em acréscimos ou decréscimos em sua qualidade de vida.

A aplicação da pesquisa mostrou que parcela significativa da população rio-branquense possui boa percepção sobre a importância do PACM, visto que o consideram como algo imprescindível.

Especificamente nesta pesquisa, uma das limitações existentes foi o fato desta ter sido conduzida dentro do parque; conseqüentemente, a VDAP e o VET refletem as preferências dos freqüentadores deste ativo. Logo, o valor social do parque não foi determinado, mas apenas o valor de uso. Os não-freqüentadores não fizeram parte da amostragem; possivelmente, as preferências desses indivíduos são diferentes das dos freqüentadores, logo, o VET atribuído ao PACM será diferente.

A aplicação do bootstrapping e da análise de risco propiciou maior confiabilidade econométrica às estimativas, em virtude da criação de um intervalo de confiança para VDAP. Posteriormente, a análise de risco possibilitou a simulação de cenários que envolviam o risco, o que contribui para determinação de um máximo e de mínimo da VDAP e do VET do PACM.

Estudos posteriores devem procurar avaliar o valor da VDAP de nãousuários e usuários, consequentemente determinando o valor social do parque. 


\section{Referências bibliográficas}

AGUIRRE, A., FARIA, D.M.C.P. Avaliação contingente de investimentos ambientais: um estudo de caso. Estudos Econômicos, São Paulo, v. 26, n. 1, p. 85-109, 1996.

BELLUZZO JR., W. Valoração de bens públicos: o método de valoração contingente. São Paulo: USP, 1995. 151 p. Dissertação (Mestrado em Economia) - Universidade de São Paulo, 1995.

BELLUZZO JR., W. Avaliação contingente para valoração de projetos de conservação e melhoria dos recursos hídricos. Pesquisa e Planejamento Econômico, Rio de Janeiro, v.29, n.1, p.113-136, 1999.

FARIA, R.C., NOGUEIRA, J.M. Método de valoração contingente: aspectos teóricos e testes empíricos. Brasília, 1998. (Mimeogr.).

FREEMAN III, A.M. The measurement of environmental and resource values. Washington: Resource for the Future, 1993.

GREENE, W.H. Econometrics analysis. 2.ed. New York: Macmillan Publishing Company, 1997. 1.075 p.

GUJARATI, N.D. Econometria básica. 3.ed. São Paulo: Makron Books, 2000. 864 p.

HADKER, N. et al. Willingness-to-pay for Borivli National Park: evidence from a contingent valuation. Ecological Economics, v. 21, p. 105-122, 1997.

HANEMANN, M.W. Welfare evaluation contingent valuation experiments with discrete responses. American Journal of Agricultural Economics, n. 66, p. 332-341, 1984.

HICKS, J.R. The generalized theory of consumer surplus. Reviews of Economics Studies, v. 13, p. 68-73.1945/6.

HICKS, J.R. The reabilitation of consumer's surplus. Reviews of Economics Studies, v. 8, p. 108-115. 1940/1. 
RIBEIRO, F.L. Avaliação contingente de danos ambientais: o caso do rio Meia Ponte em Goiânia. Viçosa: UFV, 1998. 80 p. Dissertação (Mestrado em Economia Rural) - Universidade Federal de Viçosa, 1998.

SELLAR, C., CHAVAS, J.P., STOLL, J.R. Especification of the logit model: the case of valuation of non-markets goods. Journal of Environmental Economics and Management, v. 13, p. 386-390, 1986.

SELLAR, C., STOLL, J.R., CHAVAS, J.P. Valuation of empirical measures of welfare change: a comparison of non-market techniques. Land Economics, v. 61, p. 156-175, 1985.

SEMEIA - SECRETARIA MUNICIPAL DO MEIO AMBIENTE. Informações temáticas sobre o Parque Ambiental "Chico Mendes". Rio Branco, 1996.

SILVA, R.G. Valoração do parque ambiental "Chico Mendes”, Rio Branco - Ac: Uma aplicação probabilística do método Referendum com bidding games. Viçosa: UFV, 2003. 125 p. Dissertação (Mestrado em Economia Aplicada) - Universidade Federal de Viçosa, 2003. 